\title{
Model-Based Proprioceptive State Estimation for Spring-Mass Running
}

\author{
Özlem Gür and Uluç Saranlı
}

\begin{abstract}
Autonomous applications of legged platforms will inevitably require accurate state estimation both for feedback control as well as mapping and planning. Even though kinematic models and low-bandwidth visual localization may be sufficient for fully-actuated, statically stable legged robots, they are inadequate for dynamically dexterous, underactuated platforms where second order dynamics are dominant, noise levels are high and sensory limitations are more severe. In this paper, we introduce a model based state estimation method for dynamic running behaviors with a simple spring-mass runner. By using an approximate analytic solution to the dynamics of the model within an Extended Kalman filter framework, the estimation accuracy of our model remains accurate even at low sampling frequencies. We also propose two new event-based sensory modalities that further improve estimation performance in cases where even the internal kinematics of a robot cannot be fully observed, such as when flexible materials are used for limb designs. We present comparative simulation results to establish that our method outperforms traditional approaches which rely on constant acceleration motion models and that it 1eliminates the need for an extensive and unrealistic sensor suite.
\end{abstract}

\section{INTRODUCTION}

The ability of a robot to move around and act on its environment critically depends on its ability to sense the state of both itself and its environment. Consequently, state estimation methods have been a crucial component of research on mobile robotics with a wide variety of methods developed for platforms with simple movement patterns such as wheeled robots or kinematically observable designs such as fully actuated and statically stable legged robots [9] even while moving across rough terrain [8]. However, numerous challenges still exist for accurate state estimation with robots capable of dynamically dexterous mobility since such platforms are often underactuated and rely on second-order dynamics with state components not fully observable through kinematics alone. For instance, most efficient dynamic legged gaits such as the bound, the gallop, the trot and the pronk, all incorporate substantial flight phases during which there is no ground contact and many components of the robot state cannot be observed through proprioceptive sensing. Moreover, the presence of flexible materials and compliance in a mechanism may introduce additional complications, requiring additional instrumentation and associated sources of uncertainty and unreliability [15].

A possible solution to this problem is the use of exteroceptive sensing methods such as vision or range-sensing but these are often incapable of meeting the bandwidth and accuracy requirements of feedback controllers necessary to reliably stabilize dynamic behaviors. Limited workscapes associated with external motion tracking systems [17] and the intermittent nature of GPS-based measurements in certain environments
[18] limit their utility for use with fully autonomous mobile platforms. Visual state estimation methods by themselves often do not offer sufficient measurement bandwith and accuracy and when they do, they entail high computational loads that are not feasible for autonomous operation [17]. As a consequence, a combination of both proprioceptive and exteroceptive sensors are often used within filter based sensor fusion frameworks to combine the advantages of both approaches.

In this paper, we show how the use of an accurate analytic motion model and additional cues from intermittent kinematic events can be utilized to achieve accurate state estimation for dynamic running even with a very limited sensory suite. To this end, we work with the well-established Spring-Loaded Inverted Pendulum (SLIP) model of running, illustrated in Fig. 1] [19, 6, 26], for which we consider a variety of different available sets of sensor inputs and characterize how reduced sensory information impacts performance and investigate ways in which accurate motion models and event-based state updates can be used to cope with sensory limitations.

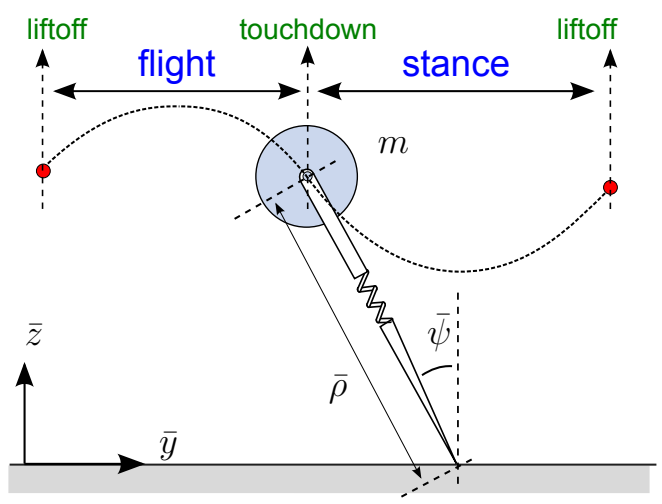

Fig. 1. The Spring-Loaded Inverted Pendulum (SLIP) model

Section $\Pi$ describes related existing work in the literature. Section [III then describes our state estimation method we propose, followed by our simulation results in Section IV Section $\nabla$ concludes the paper.

\section{RELATED WORK}

The problem of reconstructing the unknown state of a dynamical system from sensor measurements has a rich history and associated literature [27]. While this problem has wellstudied and established solution for linear systems [7], corresponding methods for nonlinear systems are diverse and varied in applicability and performance. In this context, the Extended Kalman Filter (EKF) [12, 21] has been widely used in the 
robotics community, closely followed by particle filter based methods [32] for global localization and SLAM problems [33].

One of the distinguishing aspects of legged locomotion, both from an estimation as well as a control perspective, is the invariably hybrid nature of associated dynamic models. Consequently, a number of hybrid estimation methods have been proposed for systems with multiple different phases with distinct dynamics [20] such as different leg contact configurations [31, 28] or surface conditions [30]. In any case, the consideration of structurally different hybrid states of the system substantially improves performance [15, 16]. More recent work addresses the problem of both identifying different legged gaits and using appropriate estimation algorithms and models for each to improve state estimation performance [29].

Not surprisingly, when accurate motion models are available for different modes of a hybrid dynamical system, they can be incorporated into the estimation framework to improve its performance [13, 3]. We adopt a similar approach in this paper, and focus on the Spring-Loaded Inverted Pendulum (SLIP) model that has been widely accepted and adopted both as a descriptive model and a control target [22] for running behaviors both for biological systems [6] and legged robots [19, 11, 1]. This wide acceptance motivated careful analysis of this model, leading to recently proposed approximate analytic solutions to its dynamics [24] that are sufficiently accurate to support formal inquiries on the stability of associated behaviors as well as the design of locomotion controllers [2]. The state estimation method we propose in the present paper also exploits the simplicity and accuracy of these analytical solutions for improved performance.

\section{State Estimation with the SLIP Model}

In the following sections, we will describe how continuous analytic models and event-based measurements can be used to achieve accurate state estimation for running with the SLIP model. In particular, Section III-A briefly describes the SLIP model and its dynamics, followed by a general overview of our estimation framework in Section $\amalg I I-\mathrm{B}$ and associated motion and sensor models in Sections $\amalg-\mathrm{C}$ and $\amalg I-D$ respectively.

\section{A. SLIP Model and Dynamics}

The well-known Spring-Loaded Inverted Pendulum, shown in Fig. 1 consists of a point mass $m$ connected to a passive, massless leg with linear compliance $k$. Throughout locomotion, it transitions between stance and flight phases and alternatingly experiences touchdown and liftoff events in between. During flight, the body only experiences the gravitational acceleration whereas during stance, the leg exerts spring and damper forces on the body. In order to ensure general applicability of our results, we adopt a dimensionless formulation of the system dynamics wherein we scale time variables (and derivatives with respect to time) with $\lambda:=\sqrt{\bar{\rho}_{0} / g}$ to yield $t:=\bar{t} / \lambda$ and scale all positional variables with the spring rest length as $y:=\bar{y} / \bar{\rho}_{0}$ and $z:=\bar{z} / \bar{\rho}_{0}$. The flight dynamics in these dimensionless coordinates take the form

$$
[\ddot{y}, \ddot{z}]^{T}=[0,-1]^{T},
$$

whereas stance dynamics in dimensionless polar coordinates relative to the foot location $y_{f}$ can be written as

$$
\left[\begin{array}{c}
\ddot{\psi} \\
\ddot{\rho}
\end{array}\right]=\left[\begin{array}{c}
(-2 \dot{\rho} \dot{\psi}+\sin \psi) / \rho \\
\rho \dot{\psi}^{2}-\cos \psi-\kappa(\rho-1)
\end{array}\right] .
$$

Further details of this model, its dynamics and their dimensionless formulation can be found in related literature [26, 24].

\section{B. Estimation Framework}

Following a similar approach to earlier work on hybrid state estimation for legged behaviors, we will use an Extended Kalman Filter (EKF) to process sensor readings at a frequency $f$, with the associated period defined as $T=1 / f$. In addition to these periodic readings, we will also assume that eventbased "asynchronous" readings are also available to detect touchdown and liftoff events.

Within this framework, we will rely on the presence of sufficiently accurate but possibly noisy, discrete motion and associated sensor models for a single sampling period with

$$
\begin{aligned}
\mathbf{X}_{n} & =\mathbf{g}_{i}\left(\mathbf{X}_{n-1}, \mathbf{u}_{n-1}\right)+\mathbf{w}_{i, n} \\
\mathbf{Z}_{n} & =\mathbf{h}_{j}\left(\mathbf{X}_{n}\right)+\mathbf{v}_{j, n},
\end{aligned}
$$

where $\mathbf{X}$ represents either the (dimensionless) flight state $\mathbf{X}_{f}:=[y, \dot{y}, z, \dot{z}]^{T}$ or the (dimensionless) stance state $\mathbf{X}_{s}:=$ $[\psi, \dot{\psi}, \rho, \dot{\rho}]^{T}$. The subscript $i$ indicates the presence of different motion models for different phases of the system and $j$ indexes different choices of sensor sets. The vectors $\mathbf{w}_{i, n}$ and $\mathbf{w}_{j, n}$ denote process and observation noises associated with inaccuracies in each motion and sensor model. In the rest of the paper, we do not focus on how SLIP locomotion is controlled, but assume that a suitable controller is chosen to stabilize the gait through the regulation of touchdown and liftoff leg lengths together with the touchdown leg angle to yield $\mathbf{u}_{n}$ in every step [19, 10, 24].

Similar to earlier work on hybrid state estimation, we switch between different motion and sensor models as necessary. In our application of the Extended Kalman Filter, we linearize both the motion and sensor models and use standard filter prediction and update equations [27].

\section{Motion Models for SLIP}

A common tradition in inertial guidance literature is to use a constant acceleration model during the motion prediction phase of the Kalman filter [5, 16]. In the present paper, we will use this model as a baseline for evaluating the performance of our much more accurate, model-based motion model derived through approximate analytic solutions to the SLIP dynamics. In this section, we present the details of both of these motion models to be used throughout our simulation studies.

Since flight dynamics induce simple ballistic body trajectories, we use only a single motion model for the flight phase, denoted with $\mathbf{g}_{f}$ and defined as

$$
\mathbf{X}_{f_{n}}=\left[\begin{array}{cccc}
1 & T & 0 & 0 \\
0 & 1 & 0 & 0 \\
0 & 0 & 1 & T \\
0 & 0 & 0 & 1
\end{array}\right] \mathbf{X}_{f_{n-1}}-\left[\begin{array}{c}
0 \\
0 \\
T^{2} / 2 \\
T
\end{array}\right]
$$


1) Constant Acceleration Motion Model (CAM): This commonly used motion model in the inertial navigation literature is based on the assumption that accelerations in the system, either measured through appropriate instrumentation, or predicted through the use of system dynamic equations, remain constant until the next sensor reading. In this paper, we evaluate (2) using the most recent state estimate to predict system accelerations at the beginning of every update step. We assume that accelerations remain constant in polar coordinates to yield the corresponding motion model for the stance phase as

$$
\mathbf{X}_{s_{n}}=\left[\begin{array}{cccc}
1 & T & 0 & 0 \\
0 & 1 & 0 & 0 \\
0 & 0 & 1 & T \\
0 & 0 & 0 & 1
\end{array}\right] \mathbf{X}_{s_{n-1}}+\left[\begin{array}{c}
\left(\ddot{\psi}_{n-1} T^{2}\right) / 2 \\
\ddot{\psi}_{n-1} T \\
\left(\ddot{\rho}_{n-1} T^{2}\right) / 2 \\
\ddot{\rho} T
\end{array}\right]
$$

which is already affine in the initial system states and does not need further linearization for use within the EKF framework.

Not surprisingly, the performance of this motion model degrades as the state update frequency is decreased. One of the major contributions of the present paper is to address this problem using accurate analytic solutions to SLIP trajectories with the motion model we describe next.

2) Approximate Analytic Motion Model (AAM): The stance dynamics of the SLIP model given in (2) do not admit exact analytic solutions, making it impossible to construct a motion model based on analytically computed trajectories. Fortunately, a number of very accurate approximate solutions have been proposed in the literature [10], with more recent extensions to incorporate support for damping [24].

The approximations proposed in [24] were derived considering the entire stance phase, combined with the flight phase to yield a return map to be used for controlling SLIP gaits and analyzing their stability. In our case, however, we need to support arbitrary initial conditions within the stance phase and use these approximations to predict the next state along system trajectories at the end of the corresponding sampling period. Consequently, we slightly modify solutions proposed in 24] to support different initial conditions as

$$
\begin{array}{ccc}
\psi\left(t, \mathbf{X}_{n-1}\right)= & \psi_{n-1}+Y t+Z / \omega_{0}(\sin (\phi) \\
& \left.-\sin \left(\phi+\omega_{0} t\right)\right) \\
\rho\left(t, \mathbf{X}_{n-1}\right)= & M \cos \left(\omega_{0} t+\phi\right)+F / w_{0}^{2}
\end{array}
$$

where we define

$$
\begin{aligned}
p_{\psi} & :=\rho_{n-1}^{2} \dot{\psi}_{n-1}, \omega_{0}:=\sqrt{\kappa+3 p_{\psi}^{2} / \rho_{n-1}^{4}}, \\
F & :=k-1+4 p_{\psi}^{2} / \rho_{n-1}^{3}, \\
A & :=\rho_{n-1}-F / \omega_{0}^{2}, \quad B:=\dot{\rho}_{n-1} / \omega_{0}, \\
M & :=\sqrt{A^{2}+B^{2}}, \quad \phi:=\arctan (-B / A), \\
Y & :=-2 F p_{\psi} /\left(\omega_{0}^{2} \rho_{n-1}^{3}\right)+3 p_{\psi} / \rho_{n-1}^{2}, \\
Z & :=2 M p_{\psi} / \rho_{n-1}^{3} .
\end{aligned}
$$

Note that our derivations focus on the undamped SLIP model for simplicity of presentation but they can easily be extended to the dissipative model. The motion model takes the form

$$
\mathbf{X}_{s_{n}}=\left[\begin{array}{c}
\psi\left(T, \mathbf{X}_{n-1}\right) \\
\dot{\psi}\left(T, \mathbf{X}_{n-1}\right) \\
\rho\left(T, \mathbf{X}_{n-1}\right) \\
\dot{\rho}\left(T, \mathbf{X}_{n-1}\right)
\end{array}\right],
$$

where velocity estimates are obtained through derivatives of (7) and (8). We then use straightforward analytic differentiation to linearize this map around the current estimate $\mathbf{X}_{n-1}$ and obtain EKF covariance update equations. We omit the details of this straightforward derivation for space considerations.

\section{Sensor Models}

There are numerous practical challenges in the instrumentation of dynamically dexterous, autonomous legged robots particularly when compliant members are used within the platform design [23]. Kinematic states of such flexible components require the installation, calibration and reading of strain gauges, which are usually rather noisy and fragile, especially when they are subject to harsh environmental conditions such as those experienced by legs on the platform [15]. Consequently, there is substantial practical benefit in decreasing the number of required sensory components on such platforms. In this section, we describe a number of different instrumentation alternatives for the stance phase of the SLIP model. As usual, very little information can be obtained during the flight phase, with only the leg angle measurable for the SLIP model.

1) Optimistic Sensor Model (OSM): In this paper, we use an optimistic sensor model with instrumented legs as a baseline for evaluating the performance of more limited sensory suites. In this model, we assume that the leg angle $\psi$, the leg length $\rho$ and their derivatives $\dot{\psi}$ and $\dot{\rho}$ are all measured during stance with an output vector defined as

$$
\mathbf{Z}_{o}:=\mathbf{X}_{s} \text {. }
$$

We consider this alternative "optimistic" because of substantial challenges in the reliable deployment of such sensors as evidenced by earlier implementation being constrained to operation in primarily the indoors $[15,16]$. Since the entire state vector is measurable for this sensor model, estimation performance is expected to be better than other alternatives.

2) Realistic Sensor Model (RSM): A much more realistic scenario would be when only the leg angle and its derivative are measurable. This can usually be accomplished through accurate measurement of the body angle for a mobile robot [20, 28], combined with optical encoder on hip joints. Even though flexible, composite leg designs may still introduce complications, radial compliance is usually much more dominant and orthogonal to the leg angle [19, 23, 14]. In any case, the associated output vector is defined as

$$
\mathbf{Z}_{r}:=[\psi, \dot{\psi}]^{T} .
$$

Since no information is supplied by the sensor about the radial degree of freedom, this sensor model is expected to yield low performance for the estimation of vertical degrees of freedom under traditional motion models such as the constant acceleration model. 
3) Event-based Sensor Model (ESM) : In addition to the periodic sensor measurements above, we will also introduce in this paper, a new, event-based sensor that can substantially improve estimation performance by reintroducing limited leg length measurements. In most legged robots, detection of touchdown and liftoff for individual legs is relatively straightforward, either through motor armature current measurements or dedicated contact switch components. The latter option is also capable of asynchronously triggering related software components, making it possible to decrease discretization noise arising from the periodicity of sensor readings.

The event-based sensor we introduced relies on the assumption that the leg length is known to great accuracy at the time of touchdown. The corresponding output vector is defined as

$$
\mathbf{Z}_{e}:=[\psi, \dot{\psi}, \rho]^{T},
$$

extending the realistic sensor model with a single, asynchronous reading for the leg length at touchdown. Even though a similar sensor reading can be obtained at liftoff, we only use this sensor at touchdown since damping may cause premature liftoff of the leg before it reaches full extension.

4) Time of Flight Sensor Model (ESMt): Even though the ESM sensor model supports once-per-step corrections to the leg length estimates, it provides no additional information to correct for errors in the radial velocity component during stance. Our simulation studies show that this leads to increased estimation errors and even divergence for low sampling frequencies and inaccurate motion models.

In order to address this problem, we propose to augment the ESM sensor model with time-of-flight measurements. This sensor model also benefits from the relative ease in which the touchdown and liftoff events can be asynchronously detected in practice to yield the time-of-flight $t_{f}:=t_{t d}-t_{l o}$. In contrast to the purely positional measurements provided by the sensor models described above, the measurement of the time of flight provides information on the vertical velocity at touchdown under the assumption of a known ground profile. The corresponding output vector is defined as

$$
\mathbf{Z}_{t}:=\left[\psi, \dot{\psi}, \rho, t_{f}\right]
$$

with the sensor map computing a prediction for the timeof-flight as a function of the (known but noisy) liftoff and touchdown heights and the touchdown vertical velocity $\dot{z}_{t d}$ by applying ballistic flight equations in reverse to yield

$$
t_{f}=-\dot{z}_{t d}+\sqrt{\dot{z}_{t d}^{2}-2\left(\rho_{t d} \cos \psi_{t d}-\rho_{l o} \cos \psi_{l o}\right)} .
$$

Since this function depends on the vertical touchdown velocity $\dot{z}_{t d}$, its linearization can be used for an additional EKF update at touchdown.

\section{Estimation Performance}

In this section, we present simulation studies for a comparative analysis of the proposed ESM and ESMt sensor models as well as the AAM motion model. Section IV-A describes our simulation environment and introduces performance metrics.
Section IV-C then presents a comparative study of all four sensor models, followed by an evaluation of the proposed motion model in Section IV-D under the sensor model found to have the best performance while still being practically feasible.

\section{A. Simulation Environment}

Our characterization of the estimation performance with different motion and sensor models focuses on steady-state SLIP running gaits. To this end, we first generate a large collection of SLIP trajectories with different dimensionless spring constants $k \in[25,200]$ and steady-state apex states $z \in[1.1,1.5]$ and $\dot{y}_{a} \in[0,2.5]$ under a deadbeat control strategy as proposed in [24]. These dimensionless gait and parameter ranges were selected to be consistent with both human running [4] as well as existing small robotic platforms [34, 23, 25]. In each case, the hybrid SLIP dynamics described in Section $\amalg-A$ are integrated using the ode45 solver within Matlab until at least ten steady-state steps are completed. Our evaluation of estimation performance exclusively focuses on these last ten steps since this was found to be sufficient for convergence in all simulations except those where estimation diverges. From now on, we will use the term trajectory for each run to refer these ten steps starting from touchdown.

These "ground-truth" trajectories are then uniformly sampled in time at a frequency $f$ for a realistic model of sensory acquisition in physical robot platforms. We consider the sampling frequency $f$ to be an independent variable that will be shown to have substantial impact on filter performance. Note, also, that smaller frequencies are much more desirable for practical reasons since computational resources available to autonomous mobile robots are often inadequate to support data acquisition and processing at very high frequencies. Moreover, for smaller legged platforms with smaller mechanical time constants associated with their locomotory motions (typically at $3-4 \mathrm{~Hz}$ for dynamic running), maintaining filter performance for smaller sampling frequencies becomes even more crucial for practical applicability.

Sensor readings associated with each trajectory sample are then computed using ground-truth state components. In order to ensure realistic conditions, we then add white, Gaussian noise with a signal-to-noise ratio (SNR) of 40 on these sensor readings to yield the final sensory input to the state estimator. Constant covariance matrices for each sensor model are then chosen to be consistent with this SNR value. In contrast, we compute the covariance matrix associated with the process noise $\mathbf{w}_{i, n}$ in (3) by computing prediction errors associated with the selected motion model (either CAM or AAM) starting from a large set of initial stance states, applied to a single time step of duration $T=1 / f$.

Once these uniformly sampled ground-truth data points and associated noisy sensor readings are obtained, state estimation is initialized at the first touchdown state with $\hat{\mathbf{X}}_{0}=$ $\left[\psi_{t d}, \dot{\psi}_{t d}, \rho_{t d}, \hat{\dot{\rho}}_{0}\right]$, where we assume that initial estimates for the leg angle, angular velocity and length will be relatively accurate since they are directly measurable at touchdown, whereas the initial radial velocity may have large uncertainties 
due to the lack of its direct measurements. Consequently, we also consider the initial radial velocity at touchdown, $\hat{\dot{\rho}}_{0}$ to be an independent variable which will effect the performance of various estimation algorithms. We also initialize the state covariance matrix at the initial touchdown state to be

$$
\mathbf{P}_{0}:=\left[\begin{array}{cccc}
\sigma_{\psi} & 0 & 0 & 0 \\
0 & \sigma_{\dot{\psi}} & 0 & 0 \\
0 & 0 & \sigma_{\rho} & 0 \\
0 & 0 & 0 & \sigma_{\dot{\rho}}
\end{array}\right]
$$

where $\sigma_{\psi}, \sigma_{\dot{\psi}}$ and $\sigma_{\rho}$ are chosen to be equal to the process noise of the motion model and $\sigma_{\dot{\rho}}$ in correspondence to the initial estimation error $\hat{\dot{\rho}}_{0}-\dot{\rho}_{o}$ in the initial radial velocity.
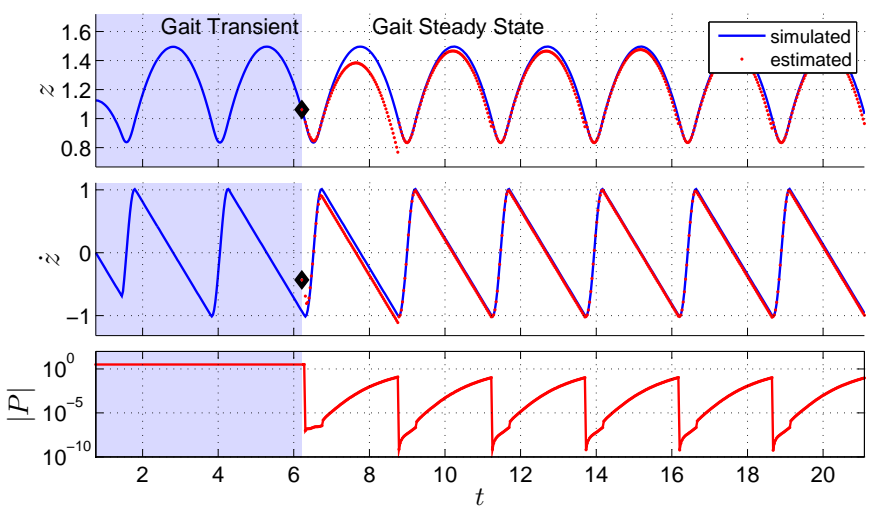

Fig. 2. An example trajectory and estimation results for vertical position (top) and velocity (middle) under our state estimation framework with the AAM motion model and the ESMt sensor model with a sampling period of $T=0.03\left(100 \mathrm{~Hz}\right.$ for $\left.\bar{\rho}_{0}=1\right)$. Estimation starts with $50 \%$ error in the radial velocity estimate (shown by a black diamond) once steady-state is reached at the end of the shaded region. States are in dimensionless coordinates and the bottom plot shows the determinant of the estimated covariance matrix $\mathbf{P}$ in logarithmic scale.

Fig. 2 illustrates an example SLIP trajectory, together with the application of our estimation algorithm with the AAM motion model and the ESMt sensor model. As evident from this figure, SLIP trajectories converge to steady-state rather quickly (in three steps) under the deadbeat controller. At the third touchdown, around $t=6.2$, the estimator starts with a relatively large, $50 \%$ error in the radial velocity and quickly converges under the motion and sensor models proposed in this paper, despite the relatively long flight phases where no sensor information is available. The corrective effect of the time-offlight update around $t=8.8$ is clearly visible in the bottom plot, illustrating the determinant of the covariance matrix.

\section{B. Convergence Criteria and Performance Metrics}

In order to assess the estimation performance of different motion and sensor model options, we define an instantaneous, normalized estimation error associated with each component of the apex state, $c \in\{\dot{y}, z, \dot{z}\}$, as

$$
E_{c}[n]:=100(\hat{c}[n]-c[n]) / \max _{n}(c[n]) .
$$

We then use a percentage error measure to characterize performance, defined as

$$
E_{T}[n]:=\left(E_{\dot{y}}[n]^{2}+E_{z}[n]^{2}+E_{\dot{z}}[n]^{2}\right)^{1 / 2} .
$$

This, in turn, yields a summary, mean square error measure for the entire duration of the 10 steady-state steps as

$$
E_{S}:=\left(\frac{1}{n_{f}-n_{0}+1} \sum_{n=n_{0}}^{n_{f}} E_{T}^{2}[n]\right)^{1 / 2}
$$

where $n_{0}$ and $n_{f}$ are the sample indices for the beginning and the end of the 10 step steady-state period. Finally, steady-state errors normalized with respect to filter confidence are captured by the Negative Log Likelihood (NLL) measure defined as

$$
\operatorname{NLL}[n]:=-\log \left(\left.\mathcal{N}(\hat{c}[n], \mathbf{P}[n])\right|_{c[n]}\right),
$$

where $\mathcal{N}$ denotes the multivariate Gaussian probability distribution function, $c[n]$ is the true system state and $\hat{c}[n]$ and $\mathbf{P}[n]$ denote the estimated mean and covariance, respectively.

It is difficult to directly use the instantaneous error measure of (18), the NLL or covariance estimates in determining EKF convergence since they show fluctuations induced by the alternating stance and flight phases. Consequently, primarily for reporting purposes within this paper, we filter the error trajectory with a fourth order zero-phase forward and backward Butterworth filter with a cutoff frequency equal to quarter of the stride frequency of the hopper to yield the filtered error trajectory $E_{F}[n]$. Using this filtered error trajectory, we report the estimator to have converged if one of the following two conditions are satisfied:

1) $E_{F}\left[n_{f}\right]<5$, meaning that the last state estimate has lower than $5 \%$ error.

2) $E_{F}\left[n_{f}\right]<100$ and $\frac{\left|E_{F}\left[n_{f}-1\right]-E_{F}\left[n_{f}-2\right]\right|}{E_{F}\left[n_{f}\right]}<0.05$, meaning that the filtered error reached steady-state and the final error magnitude is not abnormally large.

Note that the second condition is necessary for situations where the steady-state error is far from zero but still shows convergence. If neither of these conditions are satisfied, we consider the estimator to have diverged and do not incorporate the associated run in our average error results.

CAM Motion Model

\begin{tabular}{|c||c|c|c|c|}
\hline & OSM & RSM & ESM & ESMt \\
\hline \hline$E_{S}(\%)$ Mean & 2.63 & 27.61 & 18.62 & 12.49 \\
$E_{S}(\%)$ Std.Dev & 3.38 & 35.88 & 17.10 & 10.50 \\
\hline \hline Mean $N L L$ & -5.06 & 327.3 & -4.32 & -6.35 \\
\hline \hline \% Converged & 99.63 & 33.77 & 98.87 & 99.42 \\
\hline
\end{tabular}

AAM Motion Model

\begin{tabular}{|c||c|c|c|c|}
\hline$(\%)$ & OSM & RSM & ESM & ESMt \\
\hline \hline$E_{S}$ Mean & 1.62 & 11.12 & 4.08 & $\mathbf{3 . 8 3}$ \\
$E_{S}$ Std.Dev & 1.04 & 22.18 & 2.14 & $\mathbf{1 . 9 6}$ \\
\hline \hline Mean $N L L$ & -4.79 & -0.63 & -4.90 & $\mathbf{- 6 . 4 3}$ \\
\hline \hline$\%$ Converged & 99.91 & 86.69 & 99.97 & $\mathbf{9 9 . 9 7}$ \\
\hline
\end{tabular}

TABLE I

CONVERGENCE PERCENTAGES AND STATISTICS FOR THE SUMMARY MEAN SQUARE ERROR $E_{S}$ AND NLL MEASURES COMPUTED ACROSS ALL INITIAL CONDITIONS AND TRAJECTORY PARAMETERS FOR DIFFERENT MOTION AND SENSOR MODEL PAIRS (8750 RUNS EACH). 
Having defined relevant performance metrics, Table \summarizes the convergence and estimation error performance for both the CAM and AAM motion models and the four sensor models described in Section $\amalg$ II-D averaged over initial condition ranges given above, frequencies $f \in[100,1000]$ and initial radial velocity errors $\left(\hat{\dot{\rho}}_{0}-\dot{\rho}_{o}\right) / \dot{\rho}_{o} \in[-0.2,0.2]$.

\section{Comparison of Sensor Models}

We begin evaluating the performance of our estimation method by a comparison of different sensor models. The mean error figures in Table \] show that the optimistic sensor model (OSM) naturally yields the best estimation performance in general and provides an "optimal" baseline measure. Not surprisingly, the transition to the realistic sensor model (RSM) is accompanied by a sharp decrease in the convergence percentages and an associated increase in the mean estimation errors for convergent trajectories. Interestingly, however, the AAM motion model compensates for the deficiencies of the RSM sensor by enforcing conservation of angular momentum, performing corrections on leg length based on angular velocity measurements. Nevertheless, additional state updates at touchdown with the event-based sensor model (ESM) improves performance by increasing convergence percentages under the CAM motion model and improving estimation errors for the AAM motion model. Finally, the incorporation of additional time-of-flight measurements results in the best performance gains, approaching the "optimal" performance of the OSM sensor model while maintaining accuracy and convergence even for the inaccurate CAM motion model. The NLL measure also supports these observations while also taking into account a covariance-normalized interpretation of the prediction error at the last touchdown.

Performances of sensor models under different sampling frequencies is of particular interest since the choice of frequency has substantial impact on computational feasibility and practical applicability. Fig. 3illustrates the dependence of estimation performance on sampling frequency (plotted in dimensional coordinates with $\bar{\rho}_{0}=1 \mathrm{~m}$ for intuitive clarity) using the CAM motion model for all sensor models. Top and bottom plots show average convergence time and estimation errors, respectively. These results show that the RSM sensor model has the worst convergence percentages, and the ESMt sensor model yields the best estimation accuracy and convergence percentages. The increase in estimation errors with decreasing sampling frequency is due to the gradual loss of accuracy in the CAM motion model. Once again, the NLL measures are consistent with these observations and favor the ESMt sensor model. NLL figures for the RSM model fall outside the axis scale.

In summary, the convergence percentage and estimation performance of the ESMt sensor model approaches the baseline performance exhibited by the optimistic yet unrealistic OSM sensor model, yielding a feasible way in which very simple and easily deployed sensors can be used for robust state estimation. In the next section, we will show that the adoption of a more accurate motion model further improves estimation

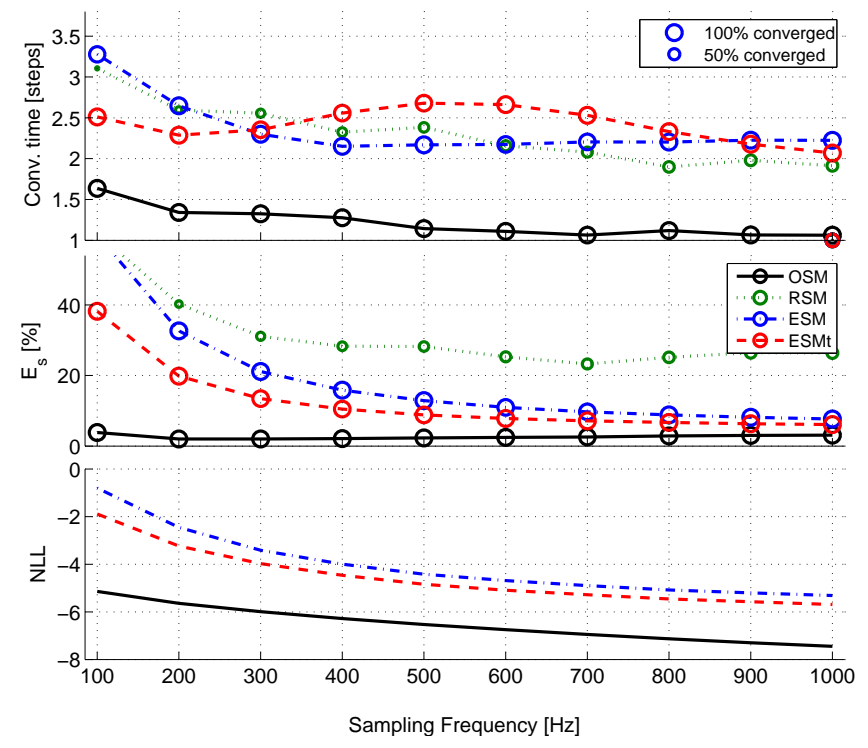

Fig. 3. Average convergence time (top), estimation errors (middle) and NLL measures (bottom) for the OSM, RSM, ESM and ESMt sensor models under the CAM motion model as a function of the (dimensional) sampling frequency. Marker sizes are proportional to convergence percentages.

performance and eliminates the degradation in accuracy with decreasing sampling frequency.

\section{Comparison of Motion Models}

Having established that using the touchdown event and timeof-flight measurements for additional Kalman updates results in substantial accuracy gains, we now focus on the motion model itself. As evident from Table I the adoption of the AAM motion model results in considerable improvement in performance with the ESM and ESMt sensor models. In the remainder of this section, we will investigate the dependence of estimation accuracy on both sampling frequency and the initial error in the radial velocity estimate for these two different motion models. As observed above, the use of the AAM motion model even helps with convergence problems associated with the RSM sensor model since it enforces conservation of angular momentum, making it possible to use angular velocity measurements to correct for errors in leg length. NLL measures for both models are close to each other, resulting from a somewhat larger covariance esimates associated with the AAM model.

Fig. 4 shows a comparison of estimation accuracy and convergence time as a function of sampling frequency with the CAM and AAM models using ESMt sensors. The most striking performance improvement is observed for lower frequencies, where assuming constant acceleration between consecutive samples produces increasingly inaccurate prediction and degraded estimation accuracy. In contrast, performance under the AAM motion model is independent of sampling frequency, which allows lower, practically feasible sampling frequencies to be chosen. A slight improvement is also observed in convergence time across all frequencies.

Finally, Fig. 5 illustrates the dependence of estimator per- 

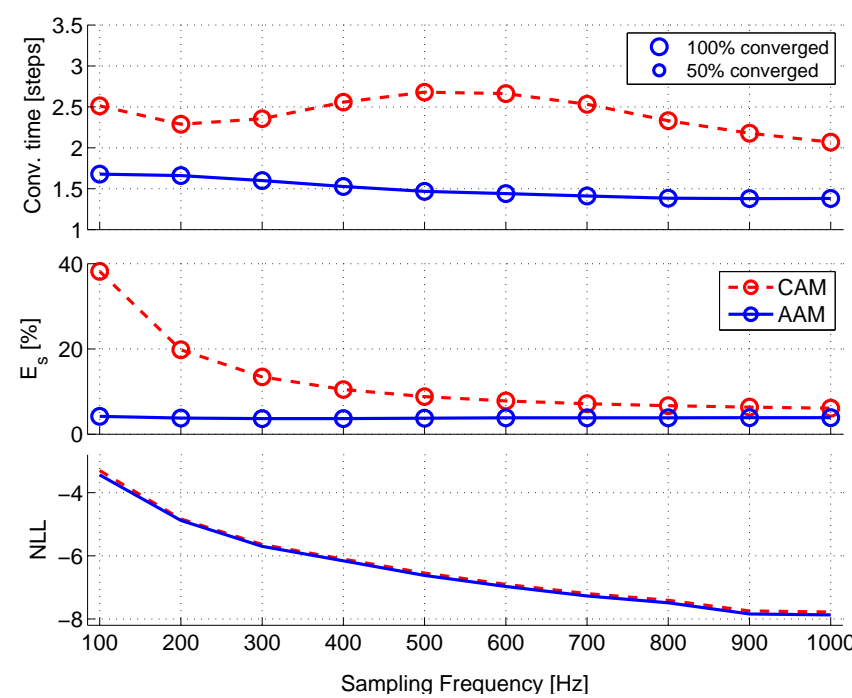

Fig. 4. Average convergence time (top), estimation errors (middle) and NLL measures (bottom) with the ESMt sensor model and two different motion models as a function of sampling frequency. Marker sizes are proportional to convergence percentages.

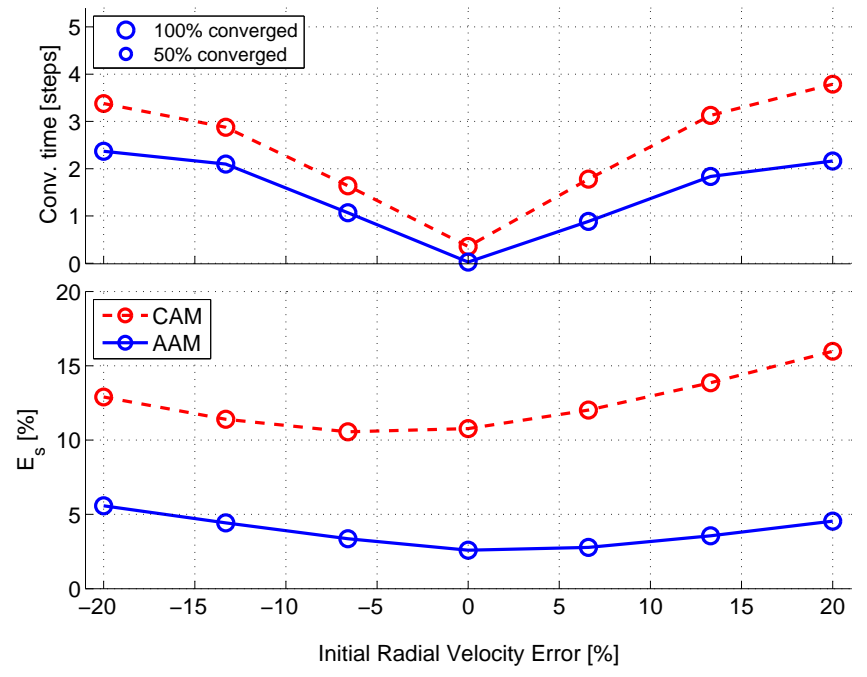

Fig. 5. Average convergence time (top) and estimation errors (bottom) with the ESMt sensor model under two different motion models as a function of initial \% estimation error in $\dot{\bar{\rho}}$. Marker sizes are proportional to convergence percentages.

formance on the initial estimation error in the radial velocity. The initial error magnitude has a small impact on steady-state estimation error, but its effect is much more pronounced on the convergence time. Once again, the AAM model consistently has better accuracy than the traditional CAM motion model and hence provides a much better state estimator design for running behaviors with the SLIP model.

\section{CONCLusions And Future Work}

In this paper, we addressed the problem of accurate state estimation for a planar spring-mass runner, a widely accepted model for running behaviors. We proposed an Extended Kalman Filter framework in which we used analytic approximations to system trajectories as to construct an accurate motion model. We also proposed two event-based, once-pertouchdown sensor inputs that are relatively simple to implement, but largely eliminate the need for complex and costly measurement of leg length and compression velocity. Our new motion model allows the filter to remain accurate even at very low sampling frequencies, while the incorporation of the eventbased sensor inputs brings estimation performance close to the best possible pallet of sensors with a fully observable state vector. In summary, the combination of our analytic motion model and the event-based sensor inputs result in average estimation errors lower than $\% 7$ and filter convergence of more than $\% 90$ across a large range of steady state running speeds, hopping heights, leg compliance and sampling frequency values, while still relying on simple sensors that are very easy to deploy on physical legged platforms.

The experimental verification of our performance results, which currently rely on simulated trajectories of the springmass runner, is pending the completion of a planarized, onelegged compliant runner platform and the extension of the motion model to incorporate damping and active torque actuation at the hip. Components necessary for these extensions to the motion model already exist in the literature [2] and are unlikely to introduce any difficulties, leaving only possible discrepancies between the SLIP model and the physical platform as potential sources of practical problems.

In the long run, we hope to apply ideas presented in this paper to the design of state estimators for more complex, dynamically dexterous multi-legged mobile platforms. Since accurate and high-bandwidth measurements of the robot state are essential to implement model-based dynamic behaviors, we expect generalizations of these ideas to be long-term enablers for achieving mobile autonomy for legged machines.

\section{ACKNOWLEDGMENTS}

This work was supported by TUBITAK, the Scientific and Technical Research Council of Turkey project 109E032 and Özlem Gür's scholarship.

\section{REFERENCES}

[1] M. Ahmadi and M. Buehler. Controlled passive dynamic running experiments with the ARL-monopod II. IEEE Trans. on Robotics, 22(5):974-986, Oct. 2006. doi: 10. 1109/TRO.2006.878935.

[2] M. M. Ankarali and U. Saranli. Stride-to-stride energy regulation for robust self-stability of a torque-actuated dissipative spring-mass hopper. Chaos, 20(3), Sep. 2010. doi: 10.1063/1.3486803.

[3] Y. Aoustin, F. Plestan, and V. Lebastard. Experimental comparison of several posture estimation solutions for biped robot rabbit. In Proc. of the IEEE Int. Conf. on Robotics and Automation, pages 1270 -1275, May 2008.

[4] Adamantios Arampatzis, Gert-Peter Briggemann, and Verena Metzler. The effect of speed on leg stiffness and joint kinematics in human running. Journal of Biomechanics, 32:1349-1353, 1999. 
[5] B. Barshan and H. F. Durrant-Whyte. Inertial navigation systems for mobile robots. IEEE Transactions on Robotics and Automation, 11(3):328-342, 1995. doi: $10.1109 / 70.388775$.

[6] R. Blickhan and R. J. Full. Similarity in multilegged locomotion: Bouncing like a monopode. J. of Comparative Physiology A: Neuroethology, Sensory, Neural, and Behavioral Physiology, 173(5):509-517, Nov. 1993. doi: 10.1007/BF00197760.

[7] Chi-Tsong Chen. Linear System Theory and Design. Saunders College Publishing, Philadelphia, PA, USA, 1984.

[8] S. Chitta, P. Vernaza, R. Geykhman, and D.D. Lee. Proprioceptive localizatilon for a quadrupedal robot on known terrain. In Proc. of the Int. Conf. on Robotics and Automation, pages 4582 -4587, Apr. 2007.

[9] J.A. Cobano, J. Estremera, and P. Gonzalez de Santos. Location of legged robots in outdoor environments. Robotics and Autonomous Systems, 56(9):751 - 761, 2008. doi: 10.1016/j.robot.2007.12.003.

[10] H. Geyer, A. Seyfarth, and R. Blickhan. Spring-mass running: simple approximate solution and application to gait stability. Journal of Theoretical Biology, 232(3): 315-328, Feb. 2005. doi: 10.1016/j.jtbi.2004.08.015.

[11] P. Gregorio, M. Ahmadi, and M. Buehler. Design, control, and energetics of an electrically actuated legged robot. Trans. on Systems, Man, and Cybernetics, Part B: Cybernetics, 27(4):626-634, August 1997. doi: 10.1109/3477.604106.

[12] R. E. Kalman. A new approach to linear filtering and prediction problems. Transactions of the ASME - Journal of Basic Engineering, 82:35-45, 1960.

[13] V. Lebastard, Y. Aoustin, F. Plestan, and L. Fridman. Absolute orientation estimation based on high order sliding mode observer for a five link walking biped robot. In Int. Workshop on Variable Structure Systems, pages $373-378$, Jun. 2006.

[14] P.-C. Lin. Proprioceptive Sensing for a Legged Robot. $\mathrm{PhD}$ thesis, Department of Mechanical Engineering, University of Michigan, Jun. 2005.

[15] P.-C. Lin, H. Komsuoglu, and D.E. Koditschek. A leg configuration measurement system for full-body pose estimates in a hexapod robot. IEEE Trans. on Robotics, 21 (3):411-422, Jun. 2005. doi: 10.1109/TRO.2004.840898.

[16] P.-C. Lin, H. Komsuoglu, and D.E. Koditschek. Sensor data fusion for body state estimation in a hexapod robot with dynamical gaits. IEEE Trans. on Robotics, 22(5): 932-943, Oct. 2006. doi: 10.1109/TRO.2006.878954.

[17] T. B. Moeslund, A. Hilton, and V. Kruger. A survey of advances in vision-based human motion capture and analysis. Computer Vision and Image Understanding, 104(2-3):90-126, 2006. doi: 10.1016/j.cviu.2006.08.002.

[18] Bradford W. Parkinson and James J. Spilker, editors. Global Positioning System. Theory and applications, volume 163-164. American Institute of Aeronautics \& Ast, 1996.
[19] M. Raibert. Legged robots that balance. MIT Press series in artificial intelligence. MIT Press, Boston, 1986.

[20] H. Rehbinder and X. Hu. Drift-free attitude estimation for accelerated rigid bodies. Automatica, 40(4):653 659, 2004. doi: 10.1016/j.automatica.2003.11.002.

[21] K. Reif and R. Unbehauen. The extended kalman filter as an exponential observer for nonlinear systems. IEEE Transactions on Signal Processing, 47(8):2324 -2328, Aug. 1999. doi: 10.1109/78.774779.

[22] U. Saranli. Dynamic Locomotion with a Hexapod Robot. $\mathrm{PhD}$ thesis, The University of Michigan, Ann Arbor, MI, Sep. 2002.

[23] U. Saranli, M. Buehler, and D. E. Koditschek. RHex: A simple and highly mobile robot. International Journal of Robotics Research, 20(7):616-631, Jul. 2001. doi: 10.1177/02783640122067570.

[24] U. Saranli, O. Arslan, M. M. Ankarali, and O. Morgul. Approximate analytic solutions to non-symmetric stance trajectories of the passive spring-loaded inverted pendulum with damping. Nonlinear Dynamics, 62(4):729-742, 2010. doi: 10.1007/s11071-010-9757-8.

[25] A. Sato and M. Buehler. A planar hopping robot with one actuator: design, simulation, and experimental results. In Proc. of the Int. Conf. on Intelligent Robots and Systems, volume 4, pages 3540-3545, Sep. 2004.

[26] W. J. Schwind. Spring Loaded Inverted Pendulum Running: A Plant Model. Phd, University of Michigan, 1998.

[27] D. Simon. Optimal State Estimation: Kalman, H Infinity, and Nonlinear Approaches. Wiley, Jun. 2006.

[28] S. P. N. Singh and K. J. Waldron. Attitude estimation for dynamic legged locomotion using range and inertial sensors. In Proc. of the Int. Conf. on Robotics and Automation, pages 1663-1668, Apr. 2005.

[29] S. P. N. Singh and K. J. Waldron. A hybrid motion model for aiding state estimation in dynamic quadrupedal locomotion. In Proc. of the Int. Conf. on Robotics and Automation, pages 4337 -4342, Apr. 2007.

[30] S. Skaff. Context Based State Estimation for Hybrid Systems with Intermittent Dynamics. Ph.d., Carnegie Mellon University, Pittsburgh, PA, Dec. 2006.

[31] S. Skaff, A. A. Rizzi, H. Choset, and P.-C. Lin. A context-based state estimation technique for hybrid systems. In Proc. of the Int. Conf. on Robotics and Automation, pages 3935-3940. IEEE, Apr. 2005.

[32] S. Thrun, D. Fox, W. Burgard, and F. Dellaert. Robust Monte Carlo Localization for Mobile Robots. Artificial Intelligence, 128(1-2):99-141, May 2001.

[33] S. Thrun, W. Burgard, and D. Fox. Probabilistic Robotics. MIT Press, 2005.

[34] G. Zeglin. The Bow Leg Hopping Robot. Ph.D., Carnegie Mellon University, October 1999. CMU-RI-TR-99-33. 